\title{
Choroidal Vascular Ischaemia
}

\author{
PIERRE AMALRIC \\ Albi, France
}

\begin{abstract}
Summary
The term 'choroidal vascular ischaemia' refers to all the choroido-retinal changes which follow an acute or chronic circulatory disorder in the arterial capillary or venous network of the choroid. However, different clinical syndromes can be observed according to the type of vessel which is occluded, the origin and rate of development of the process.

Experimentation on animals, especially on monkeys and observations on those with limited retinal pigmentation has enabled us to study the anatomy of the choroidal circulation and the changes found in choroidal vascular ischaemia following capillary embolism or thrombosis after laser. These experiments have been accompanied by histochemical, bioelectrical and genetic examination.

For the past 20 years, angiography has been used to study the choroidal circulation, although only cardiogreen and fluorescein have proved useful.
\end{abstract}

The study of the choroid started in the XVIIIth century with the vascular impregnation methods by Ruysch ${ }^{1}$ which gave a clear definition of the vascular network. Later Haller $^{2}$ and Zinn $^{3}$ differentiated between the arterial, capillary or the venous networks.

In the XIXth century, Leber, ${ }^{4}$ following a series of experiments, was able to produce the classical scheme which is still valid today. Histological studies of the uveal network in vascular sclerosis were performed and later, Coats ${ }^{5}$ and Hepburn ${ }^{6}$ were the first to publish the findings on choroidal infacts.

In vitro or postmortem examinations were not sufficient to get a perfect capillary definition. This definition became possible in 1960 when Ashton ${ }^{7}$ and Wybar $^{8}$ produced neoprene cases of the vascular network. Castro Correia ${ }^{9}$ first published pictures of characteristic chroroidal lobule in his thesis.

Since then, many studies which have had the same aim have been aided by advances in imaging techniques. ${ }^{10-14}$
A panoramic examination of the fundus using binocular ophthalmoscopy is essential for an overall study of the choroid, together with wide angle photographic retinography. The coloured filters: blue, green and infrared enable the comparison of the pictures with normal colour photographs.

Of these, red and infrared retinographies are the most helpful. However, the green filter is very useful in investigation of albinoid patients or those with a slight pigmentation. Fluorescein angiography has been the mainstay in the morphological and dynamic study of the choroidal circulation.

Although cineangiography has been given up because of the strong luminous intensity required, video angiography using the high definition camera and electronic analysis of images, represents a new and important step in the investigation of the circulation.

Unfortunately, cardiogreen which enabled the study of the big vessels is unhelpful in the investigation of the capillary choroidal cir- 


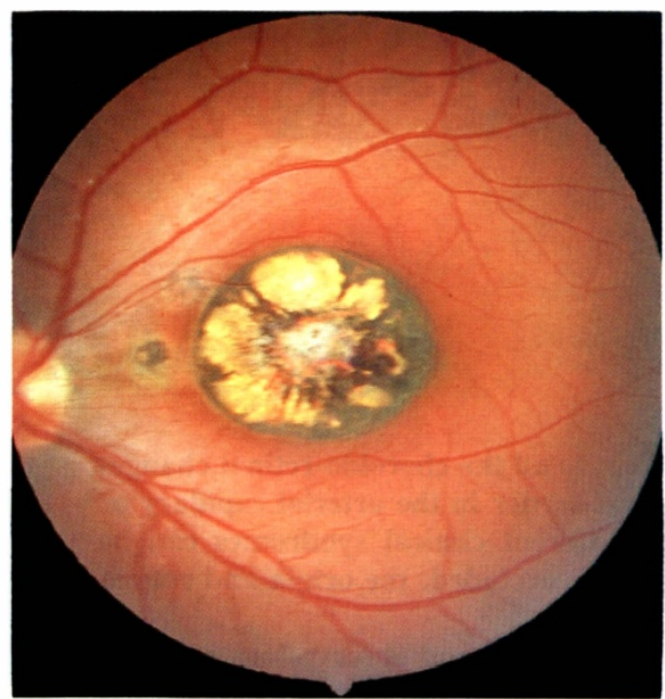

Fig. A François' rosace pattern which is typical of perifoveal arteriolar ischaemia.

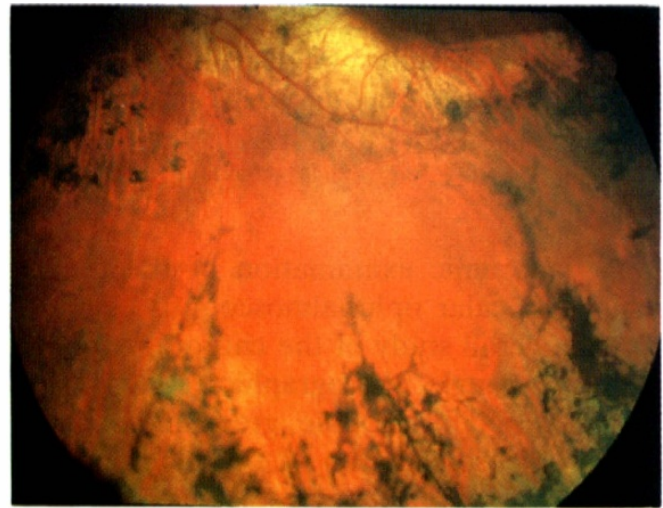

Fig. C. Triangular syndrome by traumatic ischaemia of the posterior pole.

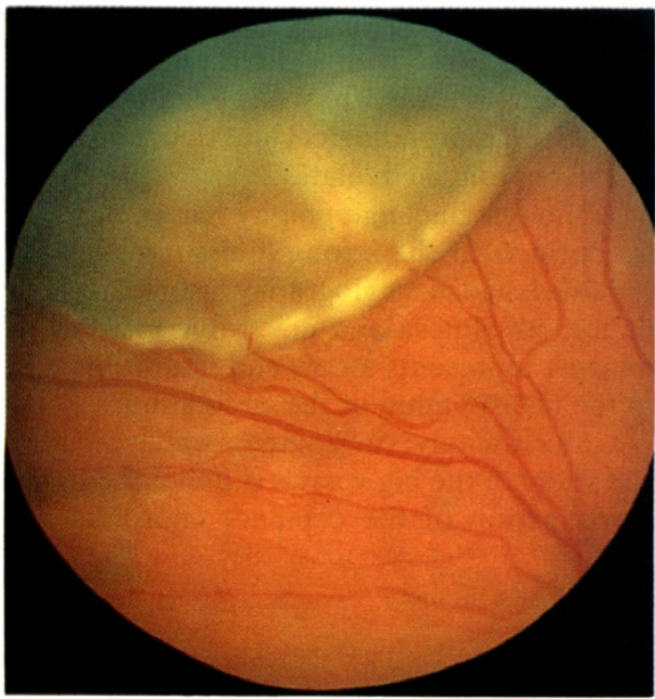

Fig. E. Pseudo melanoma by vortex thrombosis.

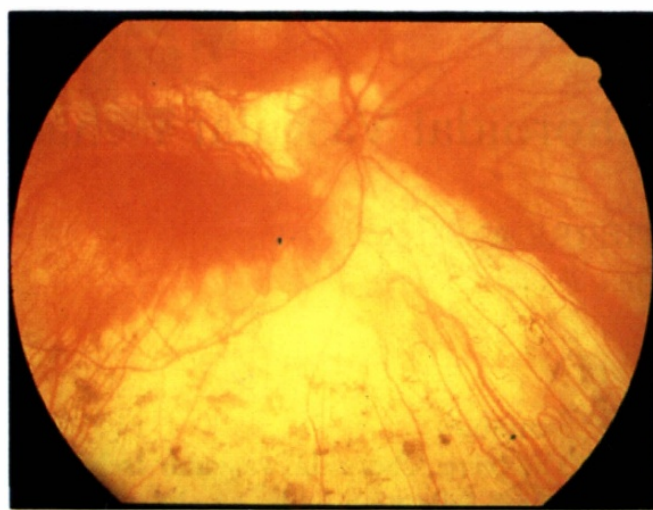

Fig. B. Congenital chorioretinal atrophy. Circulatory anomaly in the macular area.

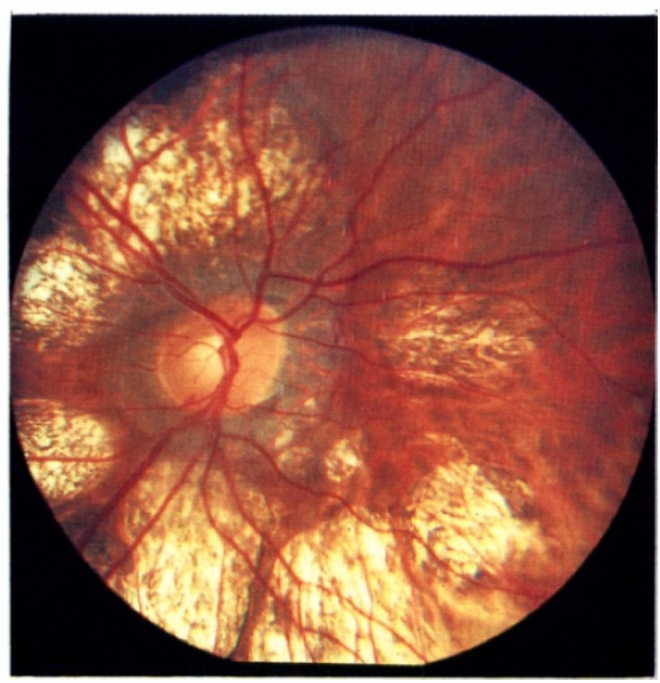

Fig. D. Circular syndrome.

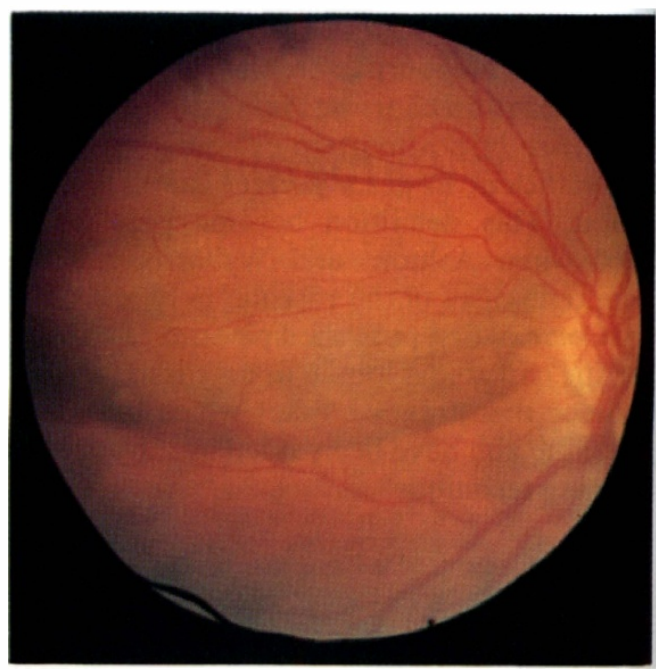

Fig. F. Same fundus 2 days after the puncture. 


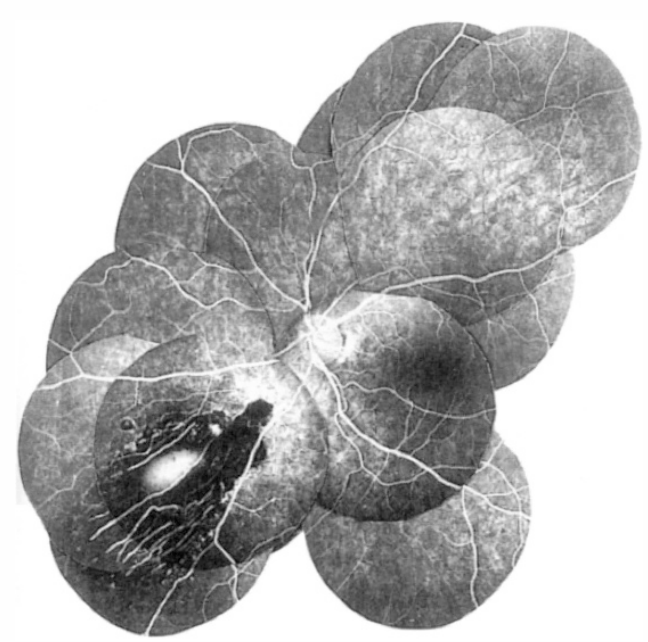

Fig. 1. Triangular syndrome.

Triangular ischaemia in the inferior nasal sector due to a toxoplasmic inflammatory lesion.

culation because of its wide fenestration which gives a rapid angiographic transit making it useless from the clinical point of view.

\section{Anatomo-clinical syndromes}

(1) The arterial syndrome

When a branch of the long posterior ciliary artery which supplies the peripheral choroid is obstructed the fluorescein discloses a triangular defect with its apex at the site of the obstruction (Fig. 1).

Hayreh's ${ }^{15}$ experimentation on monkeys showed that triangular syndromes can be observed after coagulation with laser and

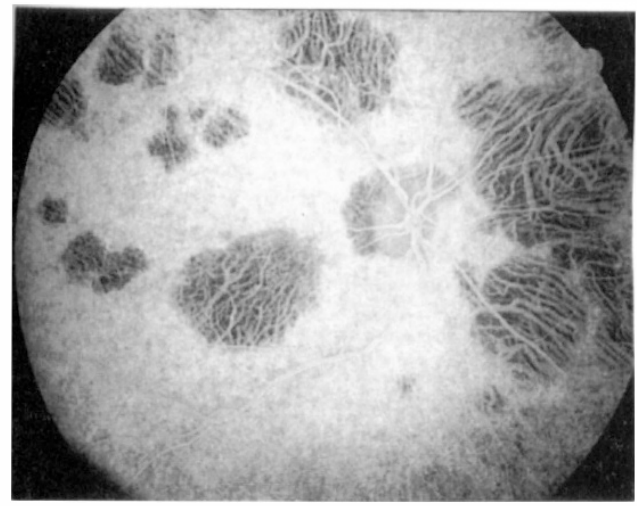

Fig. 2. Circular syndrome.

Circular ischaemia observed in a case of tapetoretinal degeneration.
Goldberg has shown that the same pattern can be noted in man after photocoagulation. The long posterior ciliary artery originates on the temporal site, lateral to the macula. Diathermy or photocoagulation in this region will induce the characteristic peripheral triangular syndrome.

The triangular syndromes caused by obstruction of the short ciliary arteries are more numerous, more irregular and are usually smaller. If they are confluent they produce the hemiatrophy of a quadrant.

As a rule, the peripheral triangular syndrome is seen in the upper quadrant between eight and two o-clock and in the inferior quadrant between four and eight o'clock and can be associated with wider peripheral circular lesions and an atrophic ring around the papilla (Figs.1-4). (Plate 1 Fig. B, C, D.)

\section{(2) The arteriolar/capillary syndromes}

Chronic or acute obstruction of the arteriolar branches or capillaries at the periphery cause atrophies which are round. These may be separated or joined; the classical pattern being the gyrate atrophy (Fig. $5 \& 6$ ).

When this type of occlusion occurs at the macula the appearances are those seen in Figures 7 and 8.

\section{(3) Choroidal ischaemia caused by venous} occlusion

The pattern produced by venous occlusion is completely different from that seen in arteriolar or arterial obstruction. Hayreh's ${ }^{16}$ experiment demonstrated that occlusion of several vortex veins produces circulatory defects which can be observed by ophthalmoscopy.

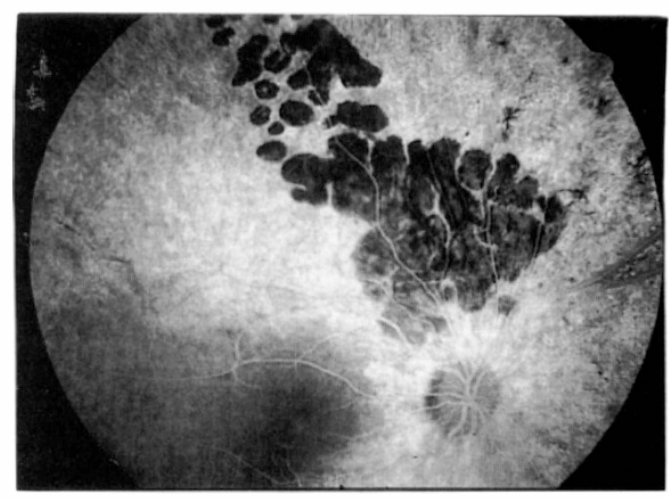

Fig. 3. Superior triangular syndrome formed by numerous circular pigmented ischaemias. 


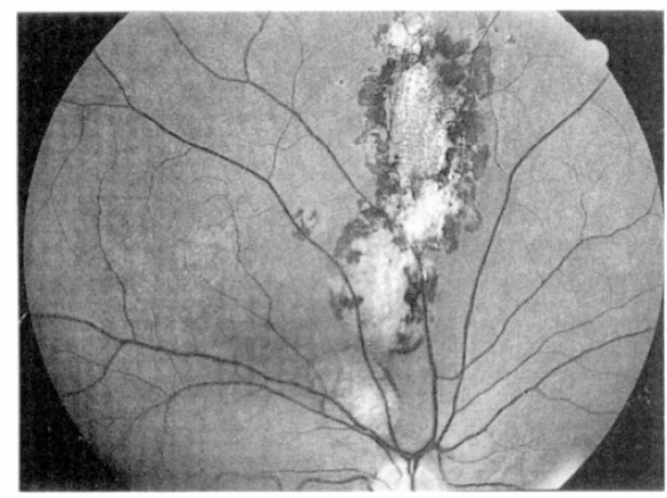

(Red-free light)

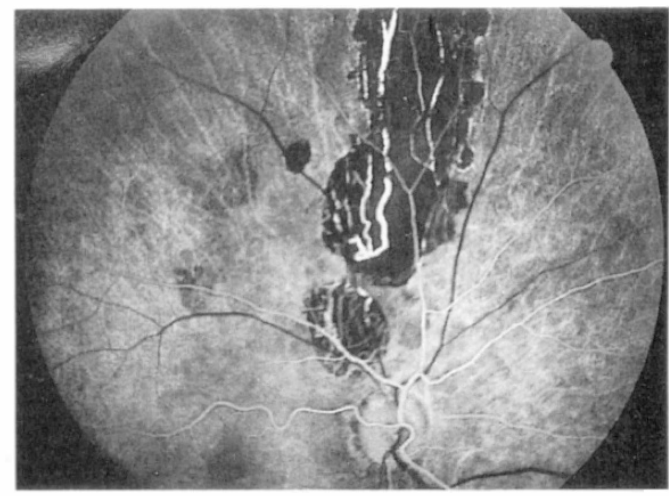

(Angriogram)

Fig. 4 Carotid thrombosis.

There appears to be a balance between the arterial and venous supply and drainage. Where there are many arteries there appear to be fewer but larger veins and vice versa. Thus, the perimacular temporal triangle is mainly arterial. The four points which correspond to the vortex veins have, on the contrary, a minimal arterial density. It also appears that if the venous density is too high
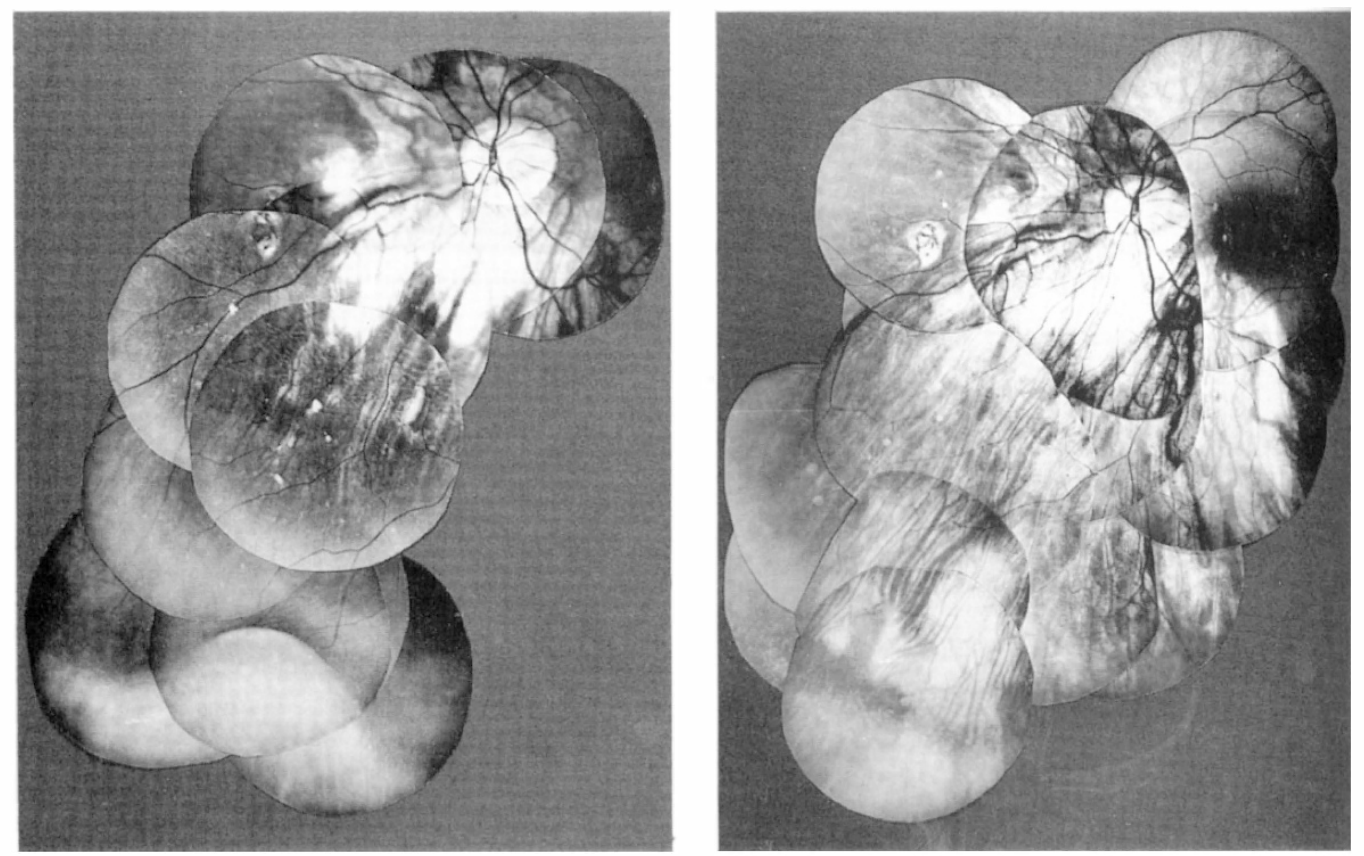

Fig. 5 Young woman suffering from an acute thrombo-phlebitis at the level of the vortex vein. Local cortico therapy. One month later, the vortex inflammation has disappeared (black).

in the macular area, some circulatory disorders may appear.

(1) The acute vortex thrombo-phlebitis leads to a chorioretinal and inflammatory detachment is often associated with local haemorrhages which respond to local and general anti-inflammatory treatment (Fig. 5).

(2) Subacute vortex thrombo-phlebitis has a 


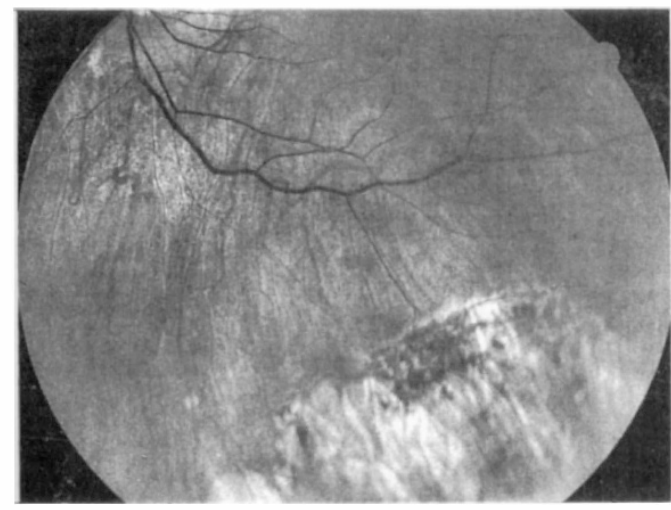

(a)

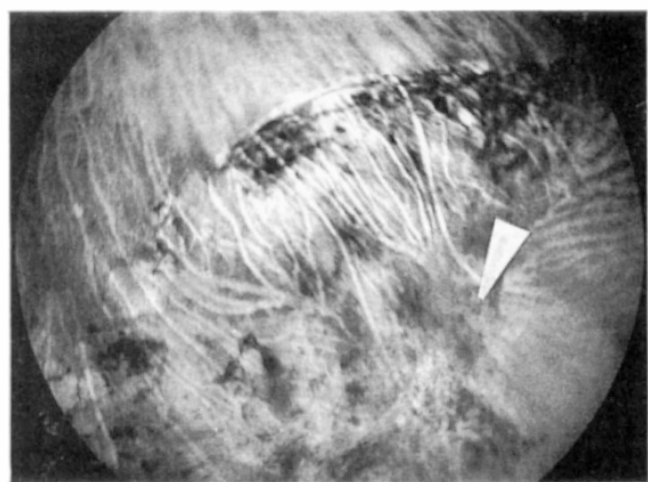

(b)

Fig. 6. Vortex occlusive syndrome. Oval pigmented scar centered on the vein. (a)-Red,(b)-Red free.

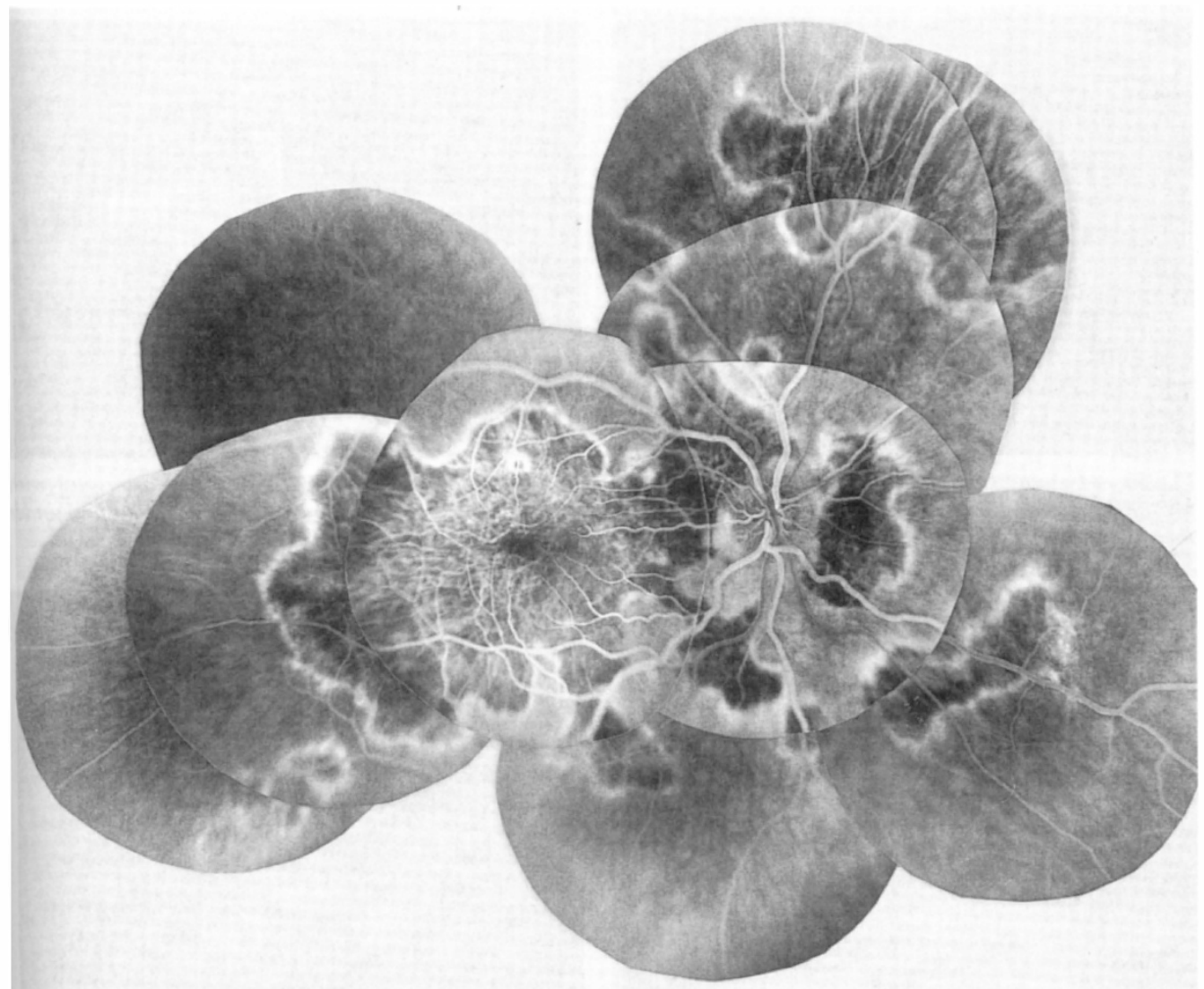

Fig. 7. Serpiginous capillary ischaemia due to the obliteration of the choriocapillaries. Star-shaped arrangement of the vessels around the fovea in the macular area. 


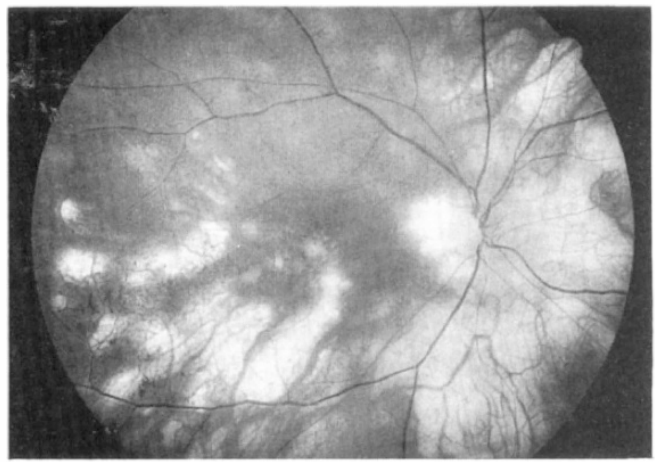

Fig. 8. Pan-retinal photocoagulation may provoke triangular syndrome by obliteration of the ciliary arteries.

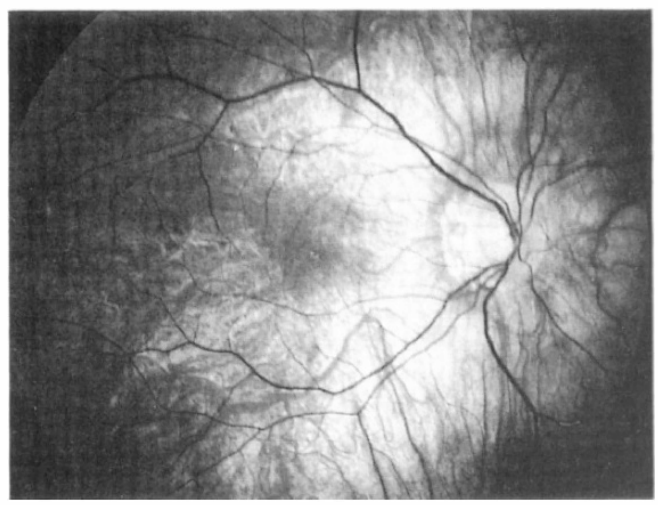

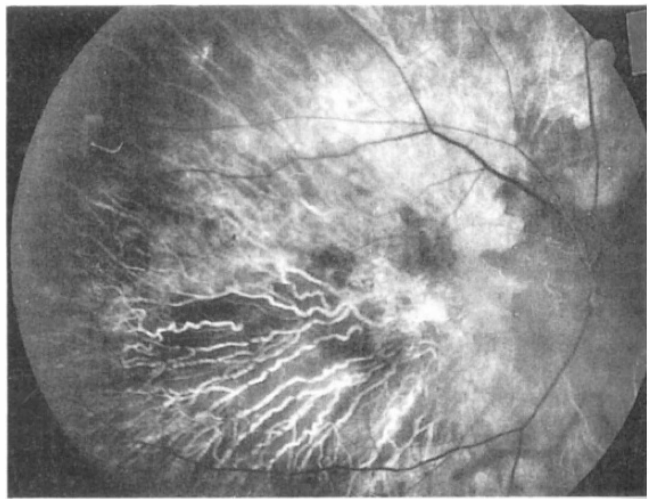

Fig. 8. Angiogram of the same patient.

Inferior triangular syndrome secondary to pan-retinal photocoagulation.

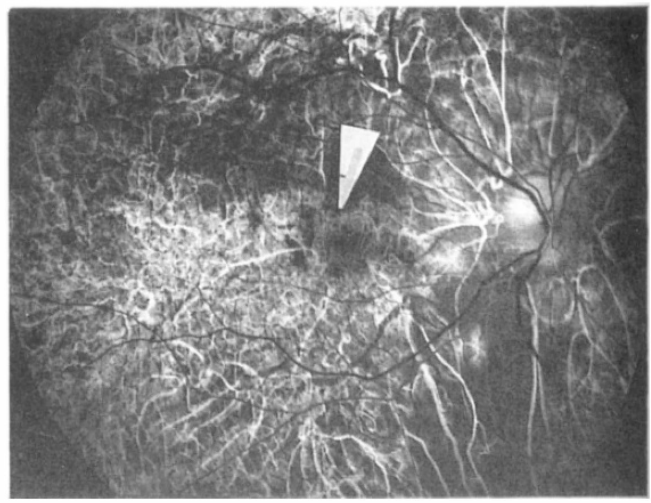

Fig. 9. Myopia with pigment rarefaction. Particular arrangement of the ciliary arteries and arterioles with visualisation of the choriocapillaris circle in the fovea. Large capillary ischaemic areas above the macular and under the papilla.
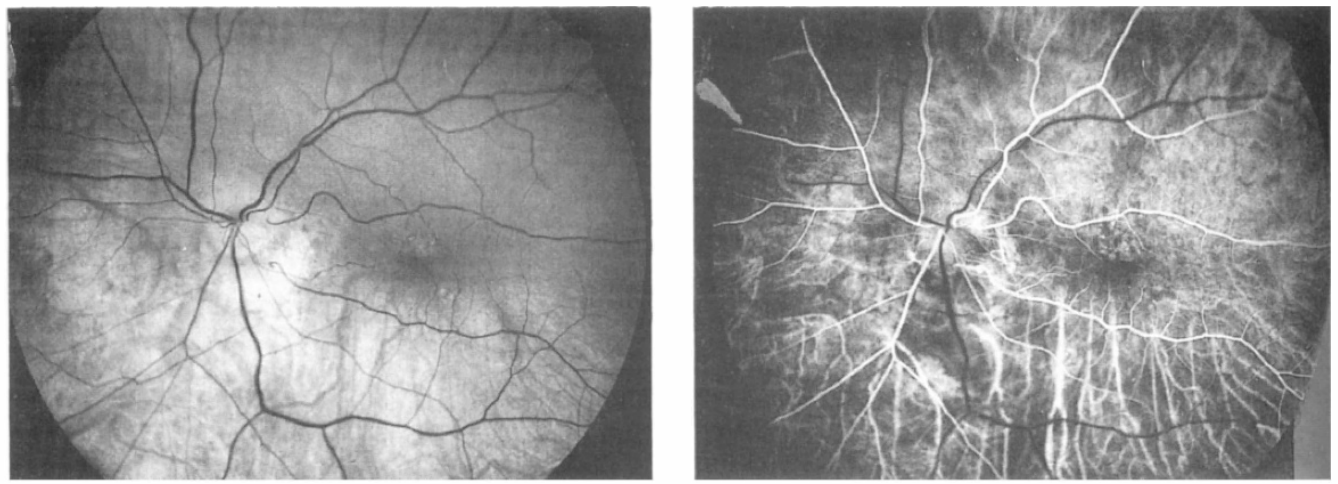

Fig. 9. Inferior choroidal hemiatrophy affecting the macular area where a pigmented dystrophy develops: Rarefaction of the choroid in all the inferior sector. 


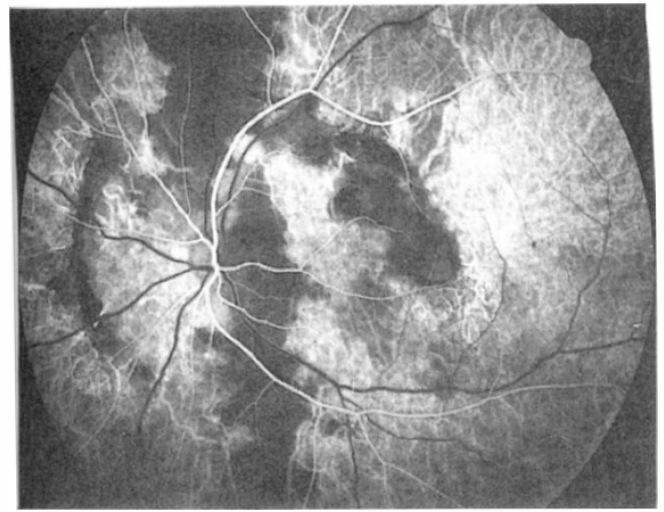

Fig. 10. Post-traumatic choroidal ischaemia. Interruption of the circulatory flow secondary to a ciliary obliteration. Blind eye.

less acute and painful development in which the pigmented lesion simulates a melanoma (Plate 1 Fig. E-F). This lesion is a choroidal haemorrhage originating from the vortex thrombosis (Fig. 6).

(3) Chronic vortex thrombo-phlebitis. In this stage venous choroidal congestion and capillary alterations are often observed e.g. in most cases of birdshot choroidopathy the big vascular trunks remain fluorescent for a long time after the injection.

(4) The capillary syndrome. When for any reason, the capillary network is obstructed, a progressive choriocapillary atrophy leading to a geographical or ser-

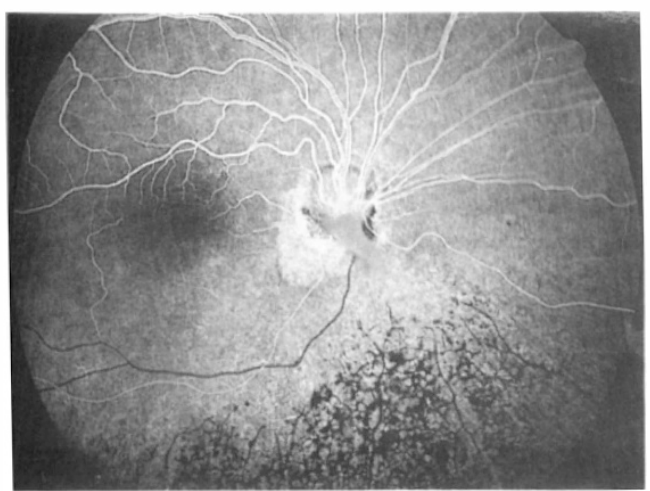

Fig. 11. Inferior triangular syndrome by endocular parasitosis which induces a retinal ischaemia emerging from the inferior branch of the central artery, and a choroidal ischaemia with an inferior triangle. Unchanged pattern for 20 years. piginous choroidopathy can be observed after the oedematous stage (Fig. 7).

Similar occlusive changes can be observed following Pan retinal photo coagulation (Fig. 8), in myopia, (Fig. 9) following trauma (Fig. 10) and some chronic infections (Fig. 11).

\section{Aetiology}

\section{Congential}

The circulatory differences between the fetus and the child largely explain the clinical patterns. We know that numerous circulatory networks disappear during the successive stages of pregnancy.

One of these is the macular choroidal circulation whose origin is from the large branches of the trunk.

\section{Choroidal circulation in the macular area}

\section{History}

As regards circulation, the macula has always been considered a unique area, being free from the blood vessels in the neuroretina; the retinal circulation converges upon the central area in successive circles. The arrangement of the subjacent choroidal circulation is much less clear and is the subject of some controversy.

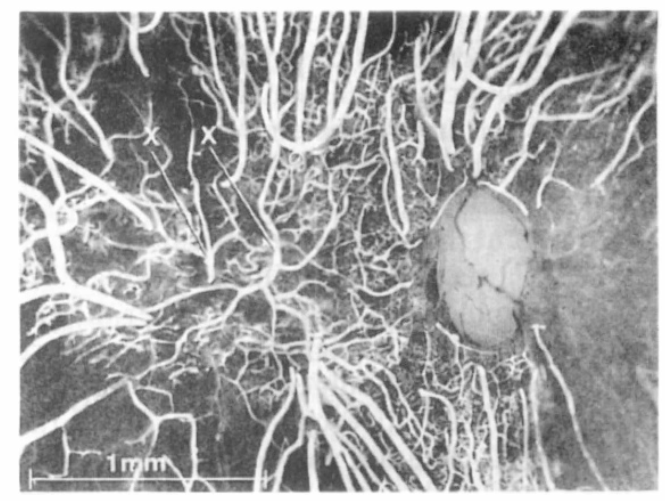

Fig. 12. We think that this big artery (x), which is particularly visible in the central part of Heimann's scheme may disappear during the formation of the fovea, in the last few months of pregnancy. Only choriocapillaris will be persistent. Our angiograms show the big vascular trunks, with their triangular arrangement which emerge around the papillo-macular triangle. Nevertheless, they are not similar to those taken by Heimann in the fetus. (Published with permission of Professor Heimann.) 


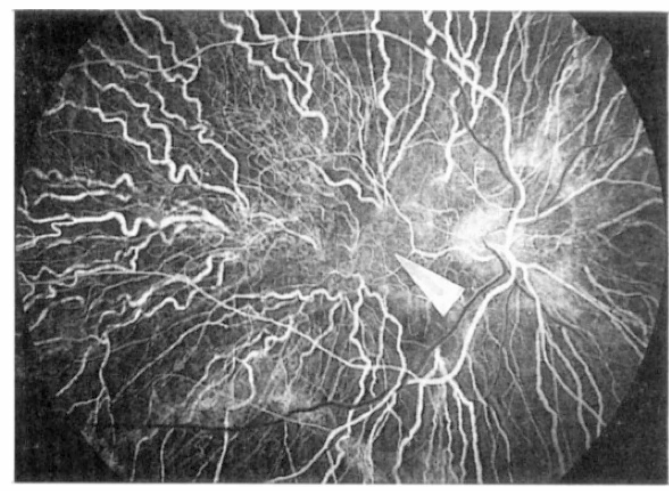

Fig. 13. Normal pattern corresponding to the arrangement of the ciliary arteries around the macula. In this area, the vascular network is not very dense; at the central point, star-shaped arrangement of the arterioles which emerge from the foveal area. This may correspond to arteriolar congenital vestiges.

For instance, Heimann ${ }^{17}$ demonstrated the existence of a specific macular artery by examining the fetus during the fifth month of pregnancy. Histological sessions showed specific vessels directed to this area (Fig. 12).

However, this notion may not seem realistic. The macular perfusion is said to be weaker in this anatomic area but this has not been confirmed by histological examinations, nor have post-mortem impregnations of the choroidal vascular network in the adult disclosed the macular artery.

Weiter ${ }^{18}$ has suggested that some of the recurrent arteries which originate in the long posterior ciliary arteries, might correspond to a macular artery. These emerge far from the macular area but an arterial branch leads back towards the fovea.

However, the syndromes seem to correspond to affection of the lobules which are centred around the foveal area rather than to arteriolar or venous changes. Each of these lobules could be the subject of infectious, vascular or degenerative conditions. Thus, the coagulation of a lobule could induce disappearance of the whole pathological vascular network as in the Rosacea of François ${ }^{19}$ which could be the expression of ischaemia of several areas which correspond to numerous parafoveal lobules. (Plate 1 Fig. A).

\section{Clinical and angiographic study}

Over the past few years the improvement of fluorescein or cardiogreen photographic techniques has enabled us to observe three major components (arterial, capillary and venous) of the choroidal capillary network in many patients with lightly pigmented fundi.

In these studies the big arteries can be shown together with their second or third divisions (Fig. 13). Capillaries can also be seen in spite of the yellow pigment (myopic, albinoid and those with a slower choroidal flow as in pigmentary retinitis), and it can be seen that choriocapillary network is extremely thin in the foveal area.

In albinoid patients with a good visual acuity it can also be seen that in the macular areas there is a central zone with thinner vascularisation which corresponds to a lighter spot. (Fig. 14). In addition large venous trunks originate in this area.

In normal individuals the macular area appears to be almost free of any first category arterial network and can only be supplied by

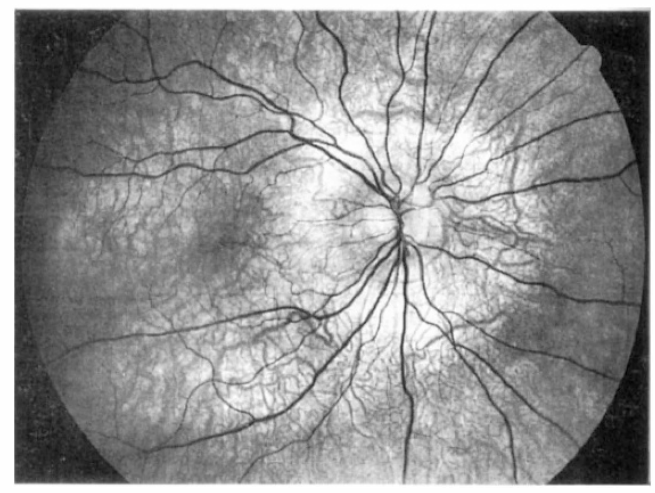

(a)

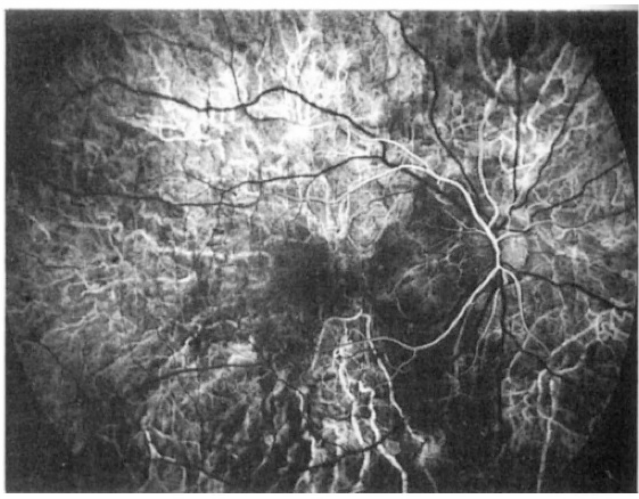

(b)

Fig. 14. Albinoid patient with preservation of a good central vision. (a) Fine visualisation of ciliary arteries and arterioles; details of theircourse. The macula is black (b). 
the vessels derived from the big vascular trunks which follow a recurrent course to the central area. These peripheral vessels are very tortuous.

The macular choroidal circulation comes from several vascular trunks and forms an initial lake rather than having a supply from an individual vessel. Thus, in the sub-foveal areas, there is virtually only one capillary network; the choroidal units develop around this foveal area.

The controversy about this area has recently been resolved. It is now agreed that the artery is not centro-lobular in all lobules as Hayreh asserted, ${ }^{20}$ but only in the central and in the mid peripheral parts of the fundus. The vein being important at the periphery, around the equator and beyond the choroidal lobules are more often centred on a vein.

\section{Embryology: hypothesis of choroidal circulation in the macular area}

Many transformations occur in the eye during fetal life. The anatomic modifications which occur very rapidly aim at organising the photographic elements which capture the light and the recording system at the level of the retina and of the superior optic tracks which will transmit the received impulses to the brain.

The vascularisation of the eye is important in the development of the globe, but at the same time, it prevents the transmission of light. Those which are likely to interface with vision such as the hyaloid arteries will regress and atrophy as does the vascular membrane which occupies the anterior chamber and the peri-lens area which disappears completely, allowing the pupil which is entirely closed during the first months of life to open in order to form a diaphragm with a variable diameter.

The choroidal system (significant in the fetus), which grows across the endocular cavity and vascularises the vitreous body as well as the posterior face of the lens also disappears leaving some residues which are more or less identifiable, either at the level of Cloquet's canal or at the level of the pupil.

Finally, the vascular retinal network which hides the fovea also disappears, although fluorescein angiography has revealed that some of these vessels sometimes persist.

From the seventh month, the macula develops in order to achieve the best visual acuity. Whilst this is happening the choroid probably undergoes a similar transformation and is reorganised in the macular and foveal areas. The big trunks which have been observed by Heimann ${ }^{21}$ in the fifth month may atrophy and disappear, enabling the development of the foveal choriocapillaris and its lobules which are perfused by some peripheral arteries explaining the patterns which we observe on the angiograms of a normal patient.

\section{References}

${ }^{1}$ Ruysch F: Opera omnia anatomico medico chirurgica. Amsterdam, J. Waesbergios, 1727-44.

${ }^{2}$ Haller H: Disputationes physico-medico anatomico-chirurgicae selectae. Neapoli B. Gessari, 1756-57.

${ }^{3}$ Zinn IG: Descriptio anatomica oculi humani. Goettingae, A. Vandenhoeck, (1780).

${ }^{4}$ Leber T: Die circulation un Eenährungsverhältnisse des auges in Saemisch T. (ed.): Graefe-Saemisch Handbuch der Gesamten Augenheilkunde. Leipzig, W. Ergelmann, vol. 2, chap. 2 (1903).

${ }^{5}$ Coats G: Posterior scleritis and infarction of the posterior ciliary arteries. Trans Ophthalmol Soc UK 1970, 27: 135 .

${ }^{6}$ Hepburn ML: The role played by the pigment and visual fields in the diagnosis of the fundus. Trans Ophthalmol Soc UK 1935, 55: 434-77.

${ }^{7}$ Ashton N: Observations on the choroidal circulation. Br J Ophthalmol 1952, 36: 465-81.

${ }^{8}$ Wybar: Vascular anatomy of the choroid in relation to selective localisation of ocular disease. $\mathrm{Br} \mathrm{J}$ Ophthalmol 1954, 38: 513-27.

${ }^{9}$ Castro Correia J: Anatomo fisiologia da coroideia. Th Fac Med Univ Porto (1958).

${ }^{10}$ Amalric Pierre: Le territoire chorio-rétinien de l'artère ciliaire longue postérieure. Bull Soc Ophthalmol France 1963, 5: 1-10.

${ }^{11}$ Amalric Pierre: Das ophthalmoskopische Bild der langen hinteren Ziliararterie. Klin Monats Für Augenheilkunde 1964, 144: 196-202.

${ }^{12}$ Amalric Pierre: L'examen clinique des artères ciliaires courtes psotérieures. Bull Soc Ophthalmol France 1968, 5/6: 1-6.

${ }^{13}$ Amalric Pierre: Veines choroïdiennes. Etat normal et pathologique. Ann Ocul 1974, 207: 161-83.

${ }^{14}$ Amalric Pierre: The choriocapillaris in the macular area. Intern Ophthalmol 1983, 6: 149-53.

${ }^{15}$ Hayreh SS: Physiological anatomy of the choroidal vasculature. Intern Ophthalmol 1983, 6: 85-93.

${ }^{16}$ Hayreh SS: Macular lesions secondary to choroidal vascular disorders. Intern Ophthalmol 1983, 6: 161-70.

${ }^{17}$ Heimann $\mathrm{K}$ and Molitor $\mathbf{J}$ : The choroidal vascular system. Acta XXIII Conc Ophthalm Kyoto 1978, 220-5.

${ }^{18}$ Weiter JJ and Ernest T: Anatomy of the choroidal vasculature. Am J Ophthalmol 1974, 78: 583-90.

${ }^{19}$ François J: Symptomatologie de la toxoplasmose. Acta I Cong Latinus Congr Ophthalmol 1953, 1: 161-311.

${ }^{20}$ Hayreh SS: Acute occlusive disorders of the choroidal vasculature. Intern Opthalmol 1983, 6: 138-9.

${ }^{21}$ Deutman AF: Acute multifocal ischaemic choroidopathy and the choriocapillaris. Intern Ophthalm ol 1983, 6: 155-60. 